\title{
Purity Determination of Three Curcuminoids Found in Ten Commercially Available Turmeric Dietary Supplements Using a Reverse Phase HPLC Method
}

\author{
Shervington $L^{*}$, Ingham $O$ and Shervington $A$
}

School of Pharmacy and Biomedical Science, University of Central Lancashire, Preston, Lancashire, UK

\begin{abstract}
The main active component present in turmeric is curcumin and is subsequently responsible for some of its therapeutic effects. Turmeric, however, contains two other active molecules, namely demethoxycurcumin (DMC) and bisdemethoxycurcumin (BDMC). Collectively, these three molecules are known as curcuminoids. Commercially available dietary supplements vary in these components. In this study the curcuminoids were analyzed using a reverse phase ion-pairing HPLC method. UV detection was used to detect the three curcuminoids at a single wavelength of $430 \mathrm{~nm}$. The validation of the method was carried to $\mathrm{ICH}$ guidelines. Good repeatability of the method was achieved at concentrations of curcumin, DMC and BDMC equivalent to 136, 148 and $162 \mu \mathrm{M}$, respectively, with R.S.D. values of $2.25,1.93$ and $1.64 \%$, respectively. Similarly, good reproducibility and linearity of the method was obtained for curcumin, DMC and BDMC. On completion of the validation, 10 commercially available turmeric dietary supplements were analyzed for their curcuminoid content. Results showed two of the turmeric dietary supplements to contain near pure curcumin. The other supplements tested contained significant amounts of DMC and BDMC. Similarly, there was a significant increase in curcuminoid content between supplements that contained turmeric extract and those that contained raw turmeric. Interestingly, supplements containing turmeric extract contained a significantly higher proportion of curcumin compared to DMC and BDMC, reflected in the ratio of curcumin: DMC: BDMC.
\end{abstract}

Keywords: $\quad$ Turmeric; Curcuminoid; Curcumin; Demethoxycurcumin; Bisdemethoxycurcumin; HPLC; Ion-pairing; Isocratic reverse phase system; Dietary supplement analysis

Abbreviations: DMC: Demethoxycurcumin; BDMC: Bisdemethoxycurcumin; PA: Peak Area; R.S.D. Relative Standard Deviation; ICH: The International Council for Harmonisation of Technical Requirements for Pharmaceuticals for Human Use.

\section{Introduction}

A variety of different curcuminoids may be derived from the south Asian spice turmeric, all with slightly different chemical compositions. The main curcuminoids contained within turmeric are curcumin, demethoxycurcumin (DMC) and bisdemethoxycurcumin (BDMC) (Figure 1). These three derivatives exhibit differing levels of therapeutic activity [1]. BDMC has been shown to exhibit a greater cytotoxicity against ovarian cancer cells compared to DMC and curcumin [2]. However, the therapeutic effects that curcuminoids generate through anti-oxidant and anti-inflammatory properties appear to be much more pronounced in curcumin and DMC compared with that of BDMC [3]. It has been shown that BDMC is a less potent inhibitor of inflammation due to its relatively reduced ability to inhibit the transcription of cyclooxygenase enzymes compared with curcumin and DMC [4].

The anti-inflammatory effects of curcumin are of particular interest and may be utilised to treat many diseases including rheumatoid arthritis, dyspepsia and stomach pain [5]. Curcumin alleviates symptoms of rhumatoid arthritis significantly better than the standard care drug, diclofenac soduim without exhibiting any of the associated adverse side effects. Remarkably, curcumin appears to have a protective effect on patients taking a combination of the two drugs and is found to show less side effects than patients taking diclofenac alone. This may be attributed to the anti-inflammatory and anti-oxidant properties of curcumin [6].

Curcumin has been shown to be unstable in aqueous solution. The molecule itself has been found to undergo rapid hydrolysis and molecular fragmentation at physiological $\mathrm{pH}$ which represents a significant limitation in its use therapeutically [7]. Stability studies conducted by Wang et al. [8], found that $90 \%$ of curcumin in an aqueous solution of phosphate buffer $(\mathrm{pH}$ 7.4) degraded within 30 minutes into multiple products including; trans-6- $\left(4^{\prime}\right.$-hydroxy- $3^{\prime}$ methoxyphenyl)-2,4-dioxo-5-hexenal, ferulic aldehyde, ferulic acid, feruloyl methane, and vanillin. This phenomenon is greatly reduced at lower $\mathrm{pH}$ values [8]. Curcumin degradation has also been analysed in the presence of both calf and human cells. The degradation of aqueous curcumin was significantly reduced in the presence of $10 \%$ foetal calf serum or human blood compared to phosphate buffer $(\mathrm{pH}$ 7.4). However, although an improvement was identified, over $50 \%$ of curcumin was found to degrade within 8 hours. Curcumin is known to be photosensitive, however photoactivated curcumin is found to be capable of much more potent anti-cancer activity [9]. Curcumin is known to be the least stable of the three curcuminoids while BDMC is known to be the most stable $[10,11]$.

The method of analysis of the curcuminoids in this study uses high performance liquid chromatography (HPLC). As a result of the curcuminoids; curcumin, DMC and BDMC possessing different polarities due to the varying number of methoxy groups, each compound exhibits a different level of interaction with the stationary phase. As a result, desorption of these compounds takes place at varying rates culminating in the separation of the three compounds. This study investigates a HPLC method for the separation of the three principle

*Corresponding author: Leroy Shervington, School of Pharmacy and Biomedical Science, University of Central Lancashire, Preston, Lancashire, PR1 2HE, UK, Tel: +4401772893519; Fax: +4401772892929; E-mail: lashervington@uclan.ac.uk

Received October 17, 2016; Accepted October 28, 2016; Published October 31, 2016

Citation: Shervington L, Ingham O, Shervington A (2016) Purity Determination of Three Curcuminoids Found in Ten Commercially Available Turmeric Dietary Supplements Using a Reverse Phase HPLC Method. Nat Prod Chem Res 4: 244 doi: 10.4172/2329-6836.1000244

Copyright: ( 2016 Shervington L, et al. This is an open-access article distributed under the terms of the Creative Commons Attribution License, which permits unrestricted use, distribution, and reproduction in any medium, provided the original author and source are credited. 
<smiles>COc1cc(/C=C/C(=O)CC(=O)/C=C/c2ccc(O)c(OC)c2)ccc1O</smiles><smiles>COc1cc(/C=C/C(=O)CC(=O)/C=C/c2ccc(O)cc2)ccc1O</smiles><smiles>O=C(/C=C/c1ccc(O)cc1)CC(=O)/C=C/c1ccc(O)cc1</smiles>

Figure 1: Chemical structure of curcuminoids.

curcuminoids found in turmeric. Repeatability, reproducibility, linearity, LOD and LOQ was determined as part of the validation protocol.

There have been numerous HPLC studies published detailing the separation of the three principle curcuminoids found in turmeric [1215]. The application of these methods is limited due to poor separation resulting in overlapping peaks and peak tailing. These limitations compromise the accuracy of quantification of these methods, reducing their effectiveness during application. Wichitnithad et al. [16] and Jadhav et al. [17] have described the best separation of the three principle curcuminoids to date.

This study aimed to accurately analyse 10 commercially available turmeric dietary supplements for their curcuminoid content using an in house established HPLC method that separates the three curcuminoids without interference. The method was based on an earlier investigation to separate 5 quinolone antibiotics by Shervington et al. [18].

\section{Materials and Methods}

\section{Chemicals}

Acetonitrile (HPLC grade $\geq 99.9 \%$ ), water (HPLC grade $\geq 99.9 \%$ ), citric acid CA (HPLC grade $\geq 99.8 \%$ ), dimethyl sulfoxide and SDS micro-pellets were purchased from Fisher Scientific. Analytical standards of curcumin ( $\geq 98 \%$ ), demethoxycurcumin $(\geq 99 \%)$, bisdemethoxycurcumin $(\geq 95 \%)$ and Tetrabutylammonium acetate (TBAA) ( $\geq 97 \%$ ) were purchased from Sigma Aldrich. Commercially available turmeric food supplements were purchased from a variety of sources as tablets or capsules and are referred to by number. Supplement 1 (450 mg turmeric extract); Supplement 2 (150 mg turmeric); Supplement 3 (400 mg turmeric); Supplement 4 (400 mg turmeric including $300 \mathrm{mg}$ turmeric extract); Supplement 5 (450 mg Turmeric); Supplement 6 (500 mg turmeric extract); Supplement 7 (500 mg turmeric); Supplement 8 (720 mg turmeric); Supplement 9 (400 mg turmeric extract); Supplement 10 (80 mg turmeric extract and $50 \mathrm{mg}$ artichoke extract).

\section{Instrumentation and HPLC method}

Instrumentation: In this study the HPLC system that was used consisted of a Jasco PU-2086 PlusIntelligent pump coupled to a Jasco UV-975 Intelligent UV / VIS detector. Data was recorded using Azur 5.0 software on a Dell Dimension 5150 computer system.

HPLC method: HPLC was carried out on an isocratic reverse phase system. The curcuminoids of interest were separated was via an ion-pairing mechanism. Samples were injected via a manual method using a Rheodyne 7725 manual injector with a $20 \mu \mathrm{L}$ loop. Samples were separated using a Waters Symmetry Shield $\mathrm{C}_{18}$ column, $(4.6 \times 250$ $\mathrm{mm}$ ), containing particles equivalent to 5 microns in size. Detection of curcumin, DMC and BDMC was determined at $430 \mathrm{~nm}$. The mobile phase consisted of a mixture of: aqueous acetonitrile-HPLC grade water $(60: 40, \mathrm{v} / \mathrm{v})$ containing tetrabutylammonium acetate $(10 \mathrm{mM})$ sodium dodecyl sulphate $(10 \mathrm{mM})$-citric acid $(25 \mathrm{mM})$. The analysis was performed at a flow rate of $1 \mathrm{~mL} / \mathrm{min}$ over a period of $15 \mathrm{mins}$ at ambient temperature.

Preparation of the mobile phase: The mobile phase was made up in batches of $1000 \mathrm{~mL}$ (600 mL acetonitrile: $400 \mathrm{~mL}$ HPLC grade water). SDS, CA and TBAA were solubilised using the 3:2 mixture of acetonitrile and water to achieve concentrations of 10,25 and $10 \mathrm{mM}$, respectively.

\section{Preparation of solvent and stock solutions}

Preparation of the solvent: The solvent used to solubilise the commercially available turmeric dietary supplements and analytical standards of curcumin, DMC and BDMC consisted of acetonitrileHPLC grade water-DMSO (35:62:3, v/v/v).

Preparation and purity of the analytical standards: Each curcuminoid standard was prepared by solubilising $10 \mathrm{mg}$ in $100 \mathrm{~mL}$ of solvent, providing solutions that equated to $271 \mu \mathrm{M}, 296 \mu \mathrm{M}$ and 324 $\mu \mathrm{M}$ of curcumin; DMC and BDMC, respectively. The purities of the analytical standards used in this research were calculated by expressing the standard's peak area as a percentage of the peaks generated by all curcuminoids within the standard. The results showed that the purity of $5 \mathrm{mg} \%$ of curcumin, DMC and BDMC were 98.7, 99.7 and $95.1 \%$, respectively.

\section{Method validation}

Following preliminary work carried out (unpublished results), the method was re-validated to confirm reliability using repeatability, reproducibility, linearity, LOD and LOQ.

Repeatability: Stock solutions $(10 \mathrm{mg} / 100 \mathrm{~mL})$ of each of the analytical standards were diluted 1:1 with solvent to give solutions that contained $5 \mathrm{mg}$ of each of the analytical standards per $100 \mathrm{~mL}$ of solvent. The resulting concentrations of $136 \mu \mathrm{M}, 148 \mu \mathrm{M}$ and 162 $\mu \mathrm{M}$ were obtained for curcumin, DMC and BDMC, respectively. Each solution was analysed six times and the peak areas (PA) recorded and the relative Standard Deviation (R.S.D.) determined.

Reproducibility: Five separate solutions of $5 \mathrm{mg} / 100 \mathrm{~mL}$ for each of the three analytical standards were prepared from the $10 \mathrm{mg} / 100$ $\mathrm{mL}$ stock solutions. Each of the five solutions were analysed twice, the average peak area recorded and the R.S.D. determined.

Linearity: The five concentrations were prepared from each of the standard stock solutions $(2,4,6,8,10 \mathrm{mg} / 100 \mathrm{~mL})$. These concentrations were then converted to $\mu \mathrm{M}$ : curcumin $54.2,108.6$, 162.6, 217.1 and $271.4 \mu \mathrm{M}$; DMC 59.2, 118.4, 177.3, 236.4 and $296 \mu \mathrm{M}$; 
BDMC 64.8, 129.7, 194.6, 259.4, 324.3 $\mu$ M. Each sample was analysed twice. Calibration curves were obtained for the three curcuminoids and the coefficients of determination $\left(\mathrm{R}^{2}\right)$ and regression equations were determined.

Lower Limit of Detection and Lower Limit of Quantification (LLOD and LLOQ): Stock solutions were diluted in a systematic manner; each diluted solution was analysed twice until the LLOD was determined. The concentration that gave a signal to noise ratio close to $10: 1$ was estimated to be the LLOQ. The LLOQ was confirmed by carrying out the determination in triplicate.

\section{Preparation and analysis of turmeric dietary supplements}

Tablet and capsule preparation: The commercially available turmeric dietary supplements analysed in this study were from capsules and tablets forms. For the capsules, the mean weight of the powder from 10 capsules was taken and the information on the packaging was used to calculate the weight of powder equivalent to $10 \mathrm{mg}$ of turmeric, taking into account the excipients. For the tablets, 10 units were powdered and the equivalent of $10 \mathrm{mg}$ of turmeric was taken (Table 1).

\begin{tabular}{|c|c|c|c|c|}
\hline $\begin{array}{c}\text { Turmeric } \\
\text { Dietary } \\
\text { Supplement }\end{array}$ & $\begin{array}{c}\text { Price } \\
\text { (pence) } \\
\text { /unit }\end{array}$ & $\begin{array}{c}\text { Average } \\
\text { weight } \mathbf{m g} \text { ) } \\
\text { (n=10) }\end{array}$ & $\begin{array}{c}\text { Turmeric content } \\
\text { per capsule or } \\
\text { tablet } \mathbf{( m g )}\end{array}$ & $\begin{array}{c}\text { Powder weight } \\
\text { equivalent to 10 mg } \\
\text { Turmeric } \mathbf{m g} \text { ) }\end{array}$ \\
\hline $\mathbf{1}$ & 30.5 & 537.53 & 450 & 11.9 \\
\hline $\mathbf{2}$ & 6.8 & 427.10 & 150 & 28.5 \\
\hline $\mathbf{3}$ & 13.9 & 448.38 & 400 & 11.2 \\
\hline $\mathbf{4}$ & 17.9 & 480.31 & 400 & 12.0 \\
\hline $\mathbf{5}$ & 6.5 & 443.20 & 450 & 10.0 \\
\hline $\mathbf{6}$ & 24.9 & 798.50 & 500 & 16.0 \\
\hline $\mathbf{7}$ & 13.6 & 490.76 & 500 & 10.0 \\
\hline $\mathbf{8}$ & 5.9 & 736.00 & 720 & 10.2 \\
\hline $\mathbf{9}$ & 52.3 & 556.60 & 400 & 13.9 \\
\hline $\mathbf{1 0}$ & 27.3 & 817.00 & 80 & 102.3 \\
\hline
\end{tabular}

Table 1: Summarizing information obtained for each of the turmeric dietary supplements.

The specified weight calculated for each of the turmeric dietary supplements were then weighed out and placed in a $50 \mathrm{~mL}$ volumetric flask, treated with $30 \mathrm{~mL}$ of solvent. The mixtures were then sonicated for 45 mins to allow sufficient time for the curcuminoids present to solubilise. The content of the flasks was then cooled and made up to the mark to afford a solution containing $20 \mathrm{mg} / 100 \mathrm{~mL}$ of turmeric. The resulting solutions were centrifuged for $20 \mathrm{mins}$ at $4000 \mathrm{G}$ to remove any remaining excipients. Samples were then diluted to a final concentration of $5 \mathrm{mg} / 100 \mathrm{~mL}$. Each step in the extraction process was validated in order to ensure that the curcuminoids were not degraded during the standard procedures.

Tablet and capsule analysis: Each of the prepared solutions of the turmeric dietary supplement samples $(5 \mathrm{mg} / 100 \mathrm{~mL})$ were analysed twice and measured against the corresponding analytical standards of curcumin, DMC and BDMC at concentrations of $5 \mathrm{mg} / 100 \mathrm{~mL}$. The curcuminoid content of each dietary supplement was then estimated.

\section{Results and Discussion}

\section{Method validation}

Repeatability: Each of the three curcuminoids were tested for repeatability as described in the method. The results for the repeatability of the curcumin, DMC and BDMC standards at 136, 148 and $162 \mu \mathrm{M}$, respectively, are shown in Table 2. For curcumin the peak areas were consistent and provided a R.S.D. of $2.25 \%$. The International Council for Harmonisation of Technical Requirements for Pharmaceuticals for Human Use (ICH) states that the maximum acceptable R.S.D. for the repeatability of a sample is $2 \%$, over six injections [19]. The R.S.D. observed for the repeatability of curcumin at $136 \mu \mathrm{M}$ is slightly over the confidence interval. However, this may be attributed to the fact that the HPLC system used was a manual injector which could account for some variation. Repeatability of DMC was carried out at $148 \mu \mathrm{M}$. The peak areas obtained were also consistent providing an R.S.D. of $1.93 \%$. Similarly, repeatability studies carried out on BDMC at $162 \mu \mathrm{M}$ were acceptable with a R.S.D. of $1.64 \%$.

\begin{tabular}{|c|c|c|}
\hline Curcuminoid & Average PA & Relative Standard Deviation (\%) $\mathbf{( n = 6 )}$ \\
\hline Curcumin $(\mathbf{1 3 6} \boldsymbol{\mu M})$ & 4155.76 & 2.25 \\
\hline DMC $(\mathbf{1 4 8} \boldsymbol{\mu M})$ & 3937.31 & 1.93 \\
\hline BDMC $(\mathbf{1 6 2} \boldsymbol{\mu M})$ & 3417.02 & 1.64 \\
\hline
\end{tabular}

Table 2: Repeatability of Curcumin, DMC and BDMC.

Reproducibility: The reproducibility of the three curcuminoids of interest involved preparing solutions of the desired concentrations of each curcuminoid, as described in the method. Reproducibility of curcumin at a concentration of $136 \mu \mathrm{M}$ showed that the average PA for five determinations was 3718 . The R.S.D. for the 5 samples was $1.95 \%$, which was within the confidence interval of $2 \%$, indicating that the method was able to produce results that were reproducible (Table 3). Reproducibility of DMC was carried out at a concentration of 148 $\mu \mathrm{M}$. The peak areas obtained were also consistent providing a R.S.D. of $1.93 \%$. Similarly, reproducibility studies carried out on BDMC at a concentration of $162 \mu \mathrm{M}$ were acceptable yielding a R.S.D. of $1.64 \%$.

\begin{tabular}{|c|c|c|}
\hline Curcuminoid & Average PA & Relative Standard Deviation (\%) $\mathbf{( n = 5 )}$ \\
\hline Curcumin $(\mathbf{1 3 6} \boldsymbol{\mu M})$ & 3717.55 & 1.95 \\
\hline DMC (148 $\boldsymbol{\mu M})$ & 4177.95 & 0.82 \\
\hline BDMC $(\mathbf{1 6 2} \boldsymbol{\mu M})$ & 3074.04 & 1.20 \\
\hline
\end{tabular}

Table 3: Summary of reproducibility data for each of the three curcuminoids.

\begin{tabular}{|c|c|c|c|}
\hline Curcuminoid & $\begin{array}{c}\text { Concentrations used } \\
\boldsymbol{\mu M}\end{array}$ & $\begin{array}{c}\text { Regression } \\
\text { equation }\end{array}$ & $\begin{array}{c}\text { Coefficient of } \\
\text { determination } \mathbf{R}^{\mathbf{2}}\end{array}$ \\
\hline Curcumin & $54,108,163,217,271$ & $\mathrm{y}=26.06 \mathrm{x}+113.60$ & 0.9989 \\
\hline DMC & $59,118,178,236,296$ & $\mathrm{y}=27.30 \mathrm{x}-20.77$ & 0.9953 \\
\hline BDMC & $65,129,195,259,324$ & $\mathrm{y}=22.53 \mathrm{x}-176.95$ & 0.9998 \\
\hline
\end{tabular}

Table 4: Summary of regression analysis for each of the three curcuminoids.

Linearity: Linearity was determined following the guidelines stipulated by the ICH in Q2R1 by testing five concentrations, (ICH, 2014). The data obtained for the linearity of the curcuminoids is summarised in Table 4 . The concentrations of curcumin that were tested were; $54,108,163,217$ and $271 \mu \mathrm{M}$. The linearity of DMC was also tested across a range of five concentrations; 59, 118, 178, 239, $296 \mu \mathrm{M}$. The linearity of BDMC was also tested across five concentrations for linearity; 65, 129, 195, 259, $324 \mu \mathrm{M}$. The regression analysis indicated a good linear relationship between response and the concentration with $\mathrm{R}^{2}$ values of $0.9989,0.9953$ and 0.9998 for curcumin, DMC and BDMC, respectively.

Lower Limit of Detection (LLOD) and Lower Limit of Quantification (LLOQ): The LLOQ and LLOD for curcumin, DMC and BDMC are summarised in Table 5. The LLOD for curcumin, DMC and BDMC was $16.9 \mathrm{nM}, 15.6 \mathrm{nM}$ and $18.0 \mathrm{nM}$, respectively (Table 5). The LLOQ for curcumin, DMC and BDMC was $33.8 \mathrm{nM}, 31.1 \mathrm{nM}$ and $32.0 \mathrm{nM}$, respectively. Acceptable repeatability for each curcuminoid was achieved over 3 determinations, verifying accurate quantification at these concentrations (Table 5). 


\begin{tabular}{|c|c|c|c|}
\hline Curcuminoid & LLOQ $\mathbf{( n M )}(\mathbf{n}=\mathbf{3})$ & RSD $(\%)$ & LLOD $(\mathbf{n M})(\mathbf{n = 2})$ \\
\hline Curcumin & 33.8 & 3.28 & 16.9 \\
\hline DMC & 31.1 & 3.00 & 15.6 \\
\hline BDMC & 32.0 & 2.83 & 18.0 \\
\hline
\end{tabular}

Table 5: Summary of LLOD and LLOQ for each of the three curcuminoids.

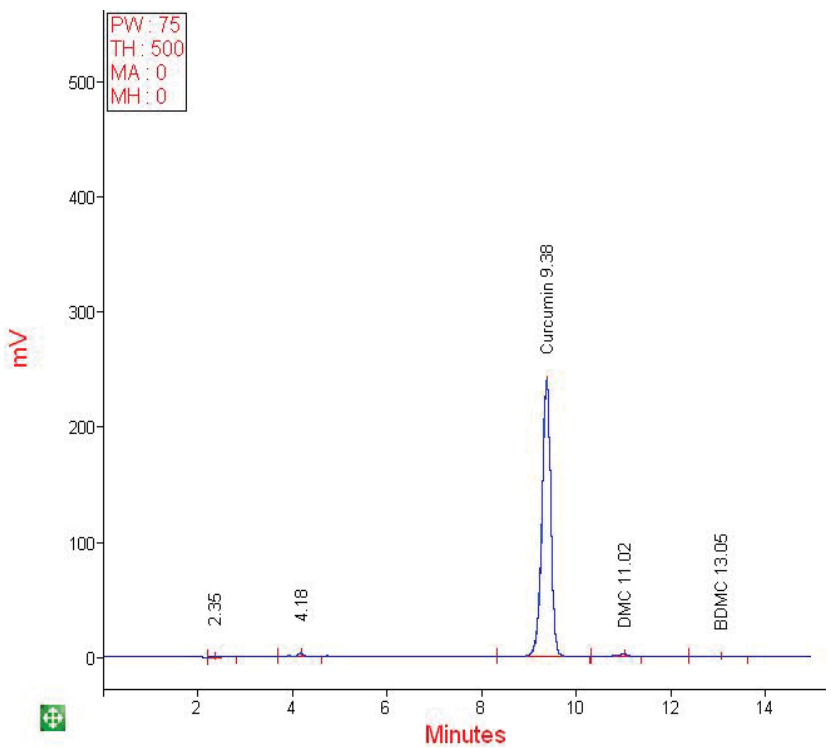

Figure 2: Typical chromatogram for analysis of curcumin analytical standard at $136 \mu \mathrm{M}$.

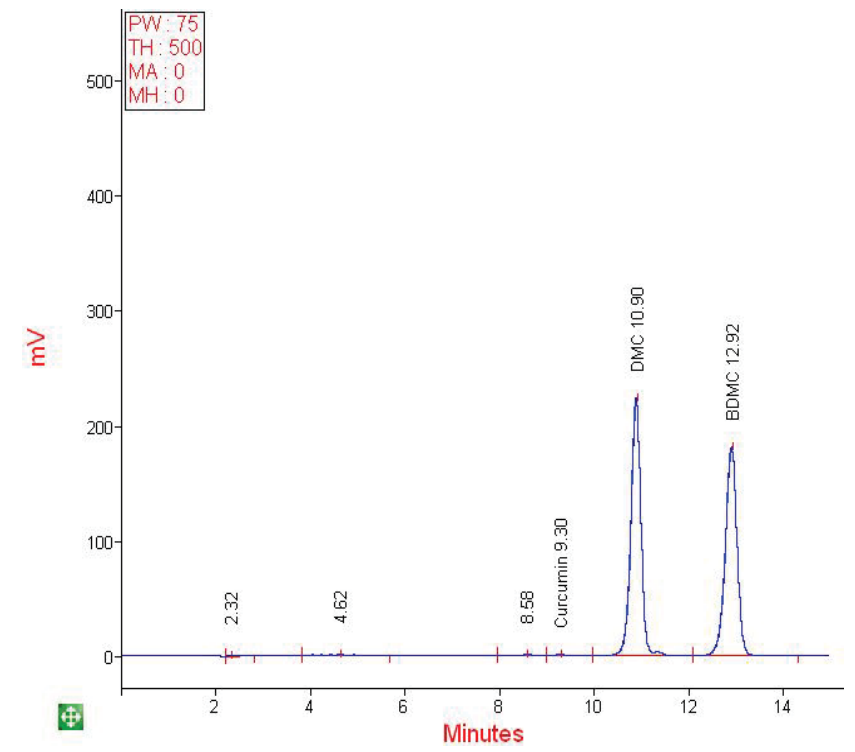

Figure 3: Typical chromatogram for DMC and BDMC analytical standards at 148 and $162 \mu \mathrm{M}$.

\section{Analysis of standards and turmeric dietary supplements}

Analysis of analytical standards: The HPLC method was validated for the analysis of curcumin, DMC and BDMC. Curcumin is the most predominant curcuminoid found in turmeric and its validation was pivotal to the analysis of the commercially available turmeric dietary supplements. A chromatogram of the curcumin analytical standard at a concentration of $136 \mu \mathrm{M}$ used to conduct the analysis is shown in Figure 2. The curcumin standard was found to be very pure ( $\geq 98.7 \%)$, containing only trace amounts of DMC and BDMC. Curcumin was eluted from the column at a retention time of approximately 9.38 mins.

Similarly, standards of BDMC and DMC were used to help validate the method. Chromatograms for DMC and BDMC at concentrations of 148 and $162 \mu \mathrm{M}$, respectively are shown in Figure 3. The DMC and BDMC standards were found to elute at a retention times of approximately 10.92 and 12.92 mins, respectively. Similar to the curcumin standard, the DMC standard was found to be very pure $(\geq 99.7 \%)$ containing only traces of curcumin and BDMC. However, although the BDMC standard was of high quality, analysis revealed it to be the least pure $(\geq 95.1 \%)$ of the analytical standards purchased containing trace amounts of DMC. The retention times for the three standards were significantly different and therefore resolved without overlap.

Turmeric dietary supplement analysis: Ten commercially available turmeric dietary supplements were analysed for their curcuminoid content. As previously discussed, the three principle curcuminoids found in turmeric are curcumin, DMC and BDMC, which differ only by the number of methoxy groups present on the aromatic rings. The major challenge faced in analysing the turmeric dietary supplements was the rather ambiguous packaging which often did not indicate the curcuminoid content of the product. Turmeric dietary supplement 6 did indicate each tablet to contain $485 \mathrm{mg}$ of curcumin but was treated the same as the other dietary supplements in order to provide results that were comparable. The curcuminoid content of each supplement was estimated by calculating the concentration of each curcuminoid and expressing this as a percentage of the respective standard.

The turmeric dietary supplements were separated into two main groups; those that contained turmeric extract and those that contained raw turmeric. Supplements 2, 3, 5, 7 and 8, containing raw turmeric, contained significantly less curcuminoids than the extracted brands. A typical chromatogram for supplement 2, shown in Figure 4, is an example that highlights the poor quality of supplements containing raw turmeric. Here, supplement 2 can be seen to exhibit a higher proportion of BDMC than DMC. As stated, BDMC has been shown to produce significantly less therapeutic antioxidant and anticancer effects when compared with curcumin and DMC, which would suggest that these supplements have a more limited therapeutic effect for the consumer. The curcuminoid ratio of non-extract supplements were found to be relatively similar with supplements $3,5,7$ and 8 containing an approximate ratio of 5:1.2:1 of curcumin, DMC and BDMC, as a result of containing the same raw material. However, the curcuminoid ratio of turmeric dietary supplement 2 was shown to be 2:1:1.3 of curcumin, DMC and BDMC, respectively, possibly as a result of the turmeric used in the supplement being obtained from a different source. However, this was difficult to confirm with any degree of confidence due to a lack of information regarding packaging.

Turmeric dietary supplements 1, 4, 6, 9 and 10 contained turmeric extract. As stated, the extract containing supplements contained a much higher curcuminoid content than the other non-extract brands. Typical chromatograms for supplements 6,9 and 10 displayed in Figures 5-7, respectively, show a superior quality of extracted brands. Figures 5-7 show extracted brands to contain a much higher proportion of curcumin and DMC which suggests these supplements carry an increased therapeutic effect for the consumer. Turmeric supplements 1 and 9 gave very similar ratios of curcumin: DMC: BDMC of 29.1:4.6:1 and 37.1:5.8:1, respectively (Table 6). Turmeric dietary supplement 4 was found to contain a ratio of curcumin, DMC and BDMC equivalent 
to 41.1: 7.6: 1 . Turmeric dietary supplements 6 and 10 contained practically pure curcumin. Chromatograms of these two supplements were similar to the analytical standard purchased from Sigma with only trace amounts of DMC and BDMC being detected (Figures 5 and 7). Interestingly, turmeric dietary supplement 10 was found to contain $112.5 \%$ curcumin when compared with the analytical standard. This finding may indicate possible issues faced in the analysis of dietary supplements as a direct result of ambiguous and possibly inaccurate packaging procedures. Brands of turmeric supplements that displayed similar ratios of curcumin, DMC and BDMC may be attributed as a result of similar turmeric extraction procedures.

Table 6 shows that all 10 tested commercially available tablets and capsules contain varied quantities of curcumin, DMC and BDMC. However, as previously mentioned, the therapeutic efficacy that curcuminoids generate through anti-oxidant and anti-inflammatory properties appear to be much more pronounced in curcumin and DMC

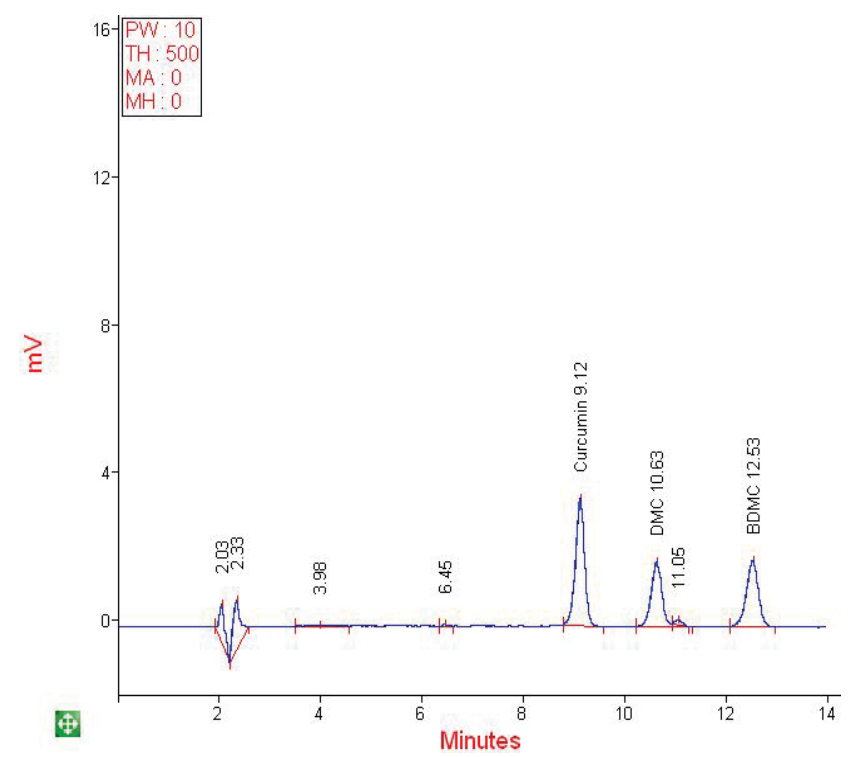

Figure 4: Typical chromatogram of turmeric dietary supplement 2.

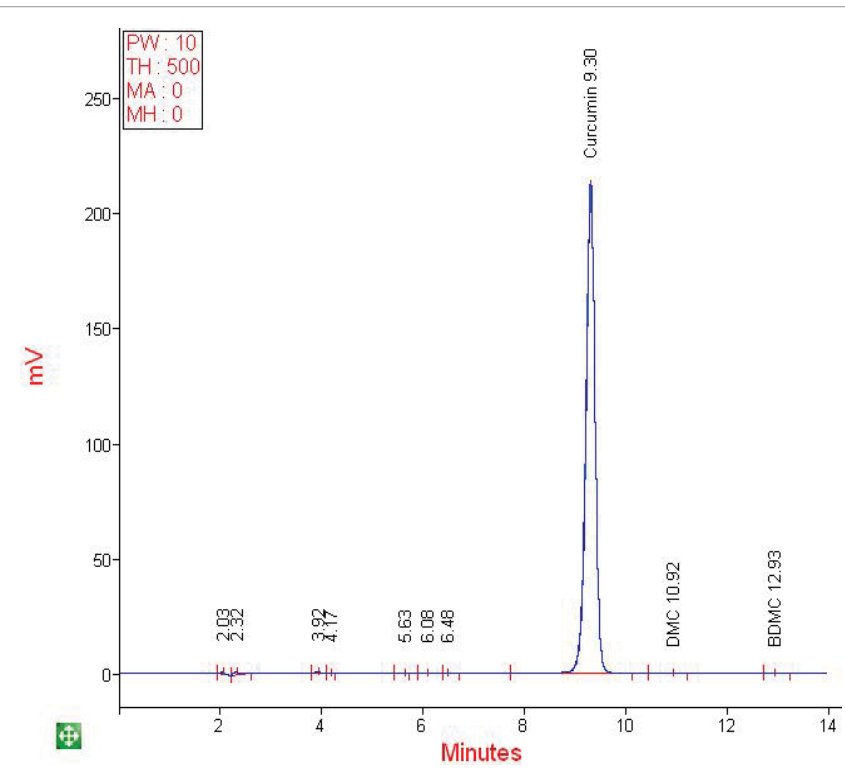

Figure 5: Typical chromatogram of turmeric dietary supplement 6.

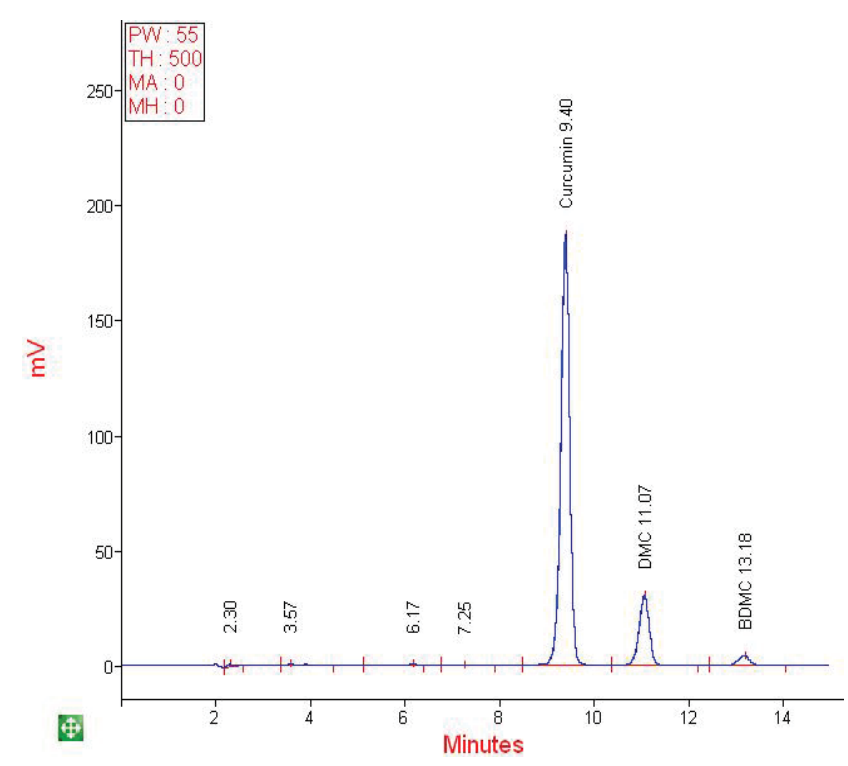

Figure 6: Typical chromatogram of turmeric dietary supplement 9.

to 41.1: 7.6: 1 . Turmeric dietary supplements 6 and 10 contained practically pure curcumin. Chromatograms of these two supplements were similar to the analytical standard purchased from Sigma with only trace amounts of DMC and BDMC being detected (Figures 5 and 7). Interestingly, turmeric dietary supplement 10 was found to contain $112.5 \%$ curcumin when compared with the analytical standard. This finding may indicate possible issues faced in the analysis of dietary supplements as a direct result of ambiguous and possibly inaccurate packaging procedures. Brands of turmeric supplements that displayed similar ratios of curcumin, DMC and BDMC may be attributed as a result of similar turmeric extraction procedures.

\begin{tabular}{|c|c|c|c|c|}
\hline $\begin{array}{c}\text { Turmeric Dietary } \\
\text { Supplement }\end{array}$ & $\begin{array}{c}\text { Curcumin } \\
\mathbf{( \% )}\end{array}$ & $\begin{array}{c}\text { DMC } \\
\mathbf{( \% )}\end{array}$ & $\begin{array}{c}\text { BDMC } \\
\mathbf{( \% )}\end{array}$ & $\begin{array}{c}\text { Ratio } \\
\text { Curcumin:DMC:BDMC }\end{array}$ \\
\hline $\mathbf{1}$ & 75.9 & 12.0 & 2.6 & $29.1: 4.6: 1$ \\
\hline $\mathbf{2}$ & 1.6 & 0.8 & 1.0 & $2: 1: 1.3$ \\
\hline $\mathbf{3}$ & 2.0 & 0.5 & 0.4 & $5: 1.3: 1$ \\
\hline $\mathbf{4}$ & 61.6 & 11.5 & 1.5 & $41.1: 7.6: 1$ \\
\hline $\mathbf{5}$ & 3.3 & 0.9 & 0.7 & $4.7: 1.3: 1$ \\
\hline $\mathbf{6}$ & 96.2 & 0.1 & 0.02 & $4810: 5: 1$ \\
\hline $\mathbf{7}$ & 2.0 & 0.6 & 0.5 & $4: 1.2: 1$ \\
\hline $\mathbf{8}$ & 1.3 & 0.3 & 0.2 & $6.5: 1.5: 1$ \\
\hline $\mathbf{9}$ & 92.8 & 14.6 & 2.5 & $37.1: 5.8: 1$ \\
\hline $\mathbf{1 0}$ & 112.5 & 0.1 & 0.02 & $5625: 5: 1$ \\
\hline
\end{tabular}

Table 6: Summary of the percentage composition of curcuminoids in commercially available turmeric dietary supplements.

Table 6 shows that all 10 tested commercially available tablets and capsules contain varied quantities of curcumin, DMC and BDMC. However, as previously mentioned, the therapeutic efficacy that curcuminoids generate through anti-oxidant and anti-inflammatory properties appear to be much more pronounced in curcumin and DMC compared to that of BDMC. Therefore, supplements 6, 9 and 10 may provide a more substantial therapeutic effect due to their curcuminoid compositions. These supplements were purchased from Life Extension (Dolcas-Biotech, New Jersey, US), HealthSpan (HealthSpan, Guernsey, UK) and Schwabe Pharma (Schwabe Pharma, Bukinghamshire, UK), respectively. 


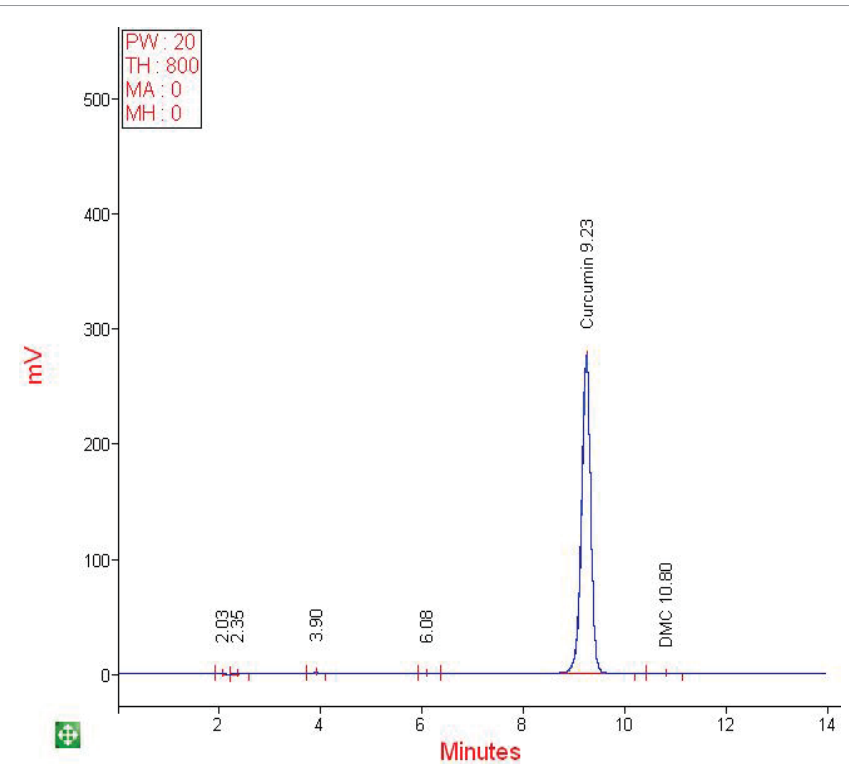

Figure 7: Typical chromatogram of turmeric dietary supplement 10.

\section{Conclusions}

In this study, a reverse phase ion-pairing HPLC method was used for separation and determination of curcumin, DMC and BDMC. The analysis was carried out in compliance with ICH guidelines yielding good repeatability, linearity and sensitivity of the method. The application of the method involved the analysis of commercially available turmeric dietary supplements. Supplements claiming to be turmeric extracts contained a significantly higher proportion of curcumin and DMC than BDMC compared to those containing raw turmeric, inferring that these supplements would generate a much more powerful therapeutic effect. We conclude that although turmeric dietary supplements do not follow FDA or MHRA regulations, with regards to quality control of their active components or their packaging, the manufacturers of these supplements have a duty to indicate the composition of their products. Greater transparency regarding the actual curcuminoid content of dietary supplements would increase greater consumer confidence.

\section{Acknowledgements}

The authors would like to express their gratitude to the School of Pharmacy and Biomedical Science for providing research funds. We also thank Roshini Matthew and Murassa Shaikh for their initial findings with regards to DMC and BDMC

\section{References}

1. Anand $P$, Thomas SG, Kunnumakkara AB, Sundaram $C$, Harikumar KB, et al (2008) Biological activities of curcumin and its analogues (Congeners) made by man and Mother Nature. Biochem Pharmacol 76: 1590-1611.

2. Syu WJ, Shen C, Don MJ, Ou JC, Lee GH, et al. (1998) Cytotoxicity of curcuminoids and some novel compounds from Curcuma zedoaria. J Nat Prod 61: $1531-1534$.

3. Ahsan H, Parveen N, Khan NU, Hadi SM (1999) Pro-oxidant, anti-oxidant and cleavage activities on DNA of curcumin and its derivatives demethoxycurcumin and bisdemethoxycurcumin. Chem Biol Interact 121: 161-175.

4. Ramsewak RS, DeWitt DL, Nair MG (2000) Cytotoxicity, antioxidant and antiinflammatory activities of curcumins I-III from Curcuma longa. Phytomed 7 : 303-308
5. Aggarwal BB, Harikumar KB (2009) Potential therapeutic effects of curcumin the anti-inflammatory agent, against neurodegenerative, cardiovascular pulmonary, metabolic, autoimmune and neoplastic diseases. The Internationa Journal of Biochemistry \& Cell Biology 41: 40-59.

6. Chandran B, Goel A (2012) A randomized, pilot study to assess the efficacy and safety of curcumin in patients with active rheumatoid arthritis. Phytother Res 26: 1719-1725.

7. Lin JK, Pan MH, Lin-Shiau SY (2000) Recent studies on the biofunctions and biotransformations of curcumin. Biofactors 13: 153-158.

8. Wang YJ, Pan MH, Cheng AL, Lin LI, Ho YS, et al. (1997) Stability of curcumin in buffer solutions and characterization of its degradation products. J Pharm Biomed Anal 15: 1867-1876.

9. Wang D, Hu J, Lv L, Xia X, Liu J, et al. (2013) Enhanced inhibitory effect of curcumin via reactive oxygen species generation in human nasopharyngeal carcinoma cells following purple-light irradiation. Oncol Lett 6: 81-85.

10. Pfeiffera E, Höhlea S, Solyomb AM, Metzlera M (2003) Studies on the stability of turmeric constituents. J Food Engineering 56: 257-259.

11. Shen L, Ji HF (2012) The pharmacology of curcumin: is it the degradation products? Trends mol Med 18: 138-144

12. Cheng J, Weijun K, Yun L, Jiabo W, Haitao W, et al. (2010) Development and validation of UPLC method for quality control of Curcuma longa Linn: Fast simultaneous quantitation of 3 curcuminoids. J Pharm Bio Anal 53: 43-49.

13. Long Y, Zhang W, Wang F, Chen Z (2013) Simultaneous determination of three curcuminoids in Curcuma longa $\mathrm{L}$. by high performance liquid chromatography coupled with electrochemical detection. J Pharm Anal 4: 325-330.

14. Jayaprakasha GK, Jagan Mohan Rao L, Sakariah KK (2002) Improved HPLC method for the determination of curcumin, demethoxycurcumin, and bisdemethoxycurcumin. J Agricultural and Food Chemistry 50: 3668-3672.

15. Syed HK, Liew KB, Loh KO, Peh KK (2015) Stability indicating HPLC-UV method for detection of curcumin in Curcuma Longa extract and emulsion formulation. Food Chemistry 170: 321-326.

16. Wichitnithad $W$, Jongaroonngamsang $N$, Pummangura $S$, Rojsitthisak $P$ (2009) A Simple Isocratic HPLC Method for the Simultaneous Determination of Curcuminoids in Commercial Turmeric Extracts. Phytochemical Analysis 20: 314-319.

17. Jadhav BK, Mahadik KR, Paradkar AR (2007) Development and Validation of Improved Reversed Phase-HPLC Method for Simultaneous Determination of Curcumin, Demethoxycurcumin and Bis-Demethoxycurcumin Chromatographia 65: 483-488.

18. Shervington LA, Abba M, Hussain B, Donnelly J (2005) The simultaneous separation and determination of five quinolone antibiotics using isocratic reversed-phase HPLC: application to stability studies on an ofloxacin tablet formulation. J Pharm Biomed Anal 39: 769-775.

19. ICH (2014) Validation of Analytical Procedures: Text and Methodology (Q2R1). 